\title{
RESEARCH HIGHLIGHT RNA and the PIEZO force sensor
}

\author{
David J. Beech (D) and Laeticia Lichtenstein (D) \\ Cell Research (2020) 30:829-830; https://doi.org/10.1038/s41422-020-00413-5
}

\begin{abstract}
The discovery of the PIEZO force-sensing ion channels was an important advance in recent years, leading to exciting new knowledge and fields of inquiry. An interesting article published by Sugisawa et al. in Cell unexpectedly suggests PIEZO1 channels as RNA rather than force sensors in enterochromaffin cells, with important implications for microbiome response and gut motility mediated by $\mathbf{5}-\mathrm{HT}$.
\end{abstract}

Sugisawa et al.'s extensive work on mice and cultured cells has led them to proposals that could profoundly change thinking about intestinal biology and how RNA is sensed. ${ }^{1}$ At the heart of their work is a protein called PIEZO1, which first came to prominence a decade ago as one of two unusual membrane proteins (PIEZO1 and PIEZO2). These proteins separately form large $\mathrm{Ca}^{2+}$-permeable non-selective cation channels with remarkable abilities to sense and respond to mechanical forces such as membrane stretch ${ }^{2-5}$ (Fig. 1a). After finding evidence of PIEZO1 expression in intestinal epithelium, Sugisawa et al. generated mice in which PIEZO1 was disrupted in epithelial chemosensory cells. ${ }^{1}$ In these mice they found slower gut transit times and protection against features of colitis in a model of this disease. 5-HT (serotonin) was considered as a potential intermediate. Consistent with this idea, serum and gut 5-HT were reduced in the PIEZO1disrupted mice, most likely because of reduced expression of the gene encoding tryptophan hydroxylase-1 (Tph1), the rate-limiting enzyme for 5-HT production.

A striking feature of this new paper ${ }^{1}$ is the authors' observation that Tph1 expression was induced by cyclical stretch via a mechanism that was PIEZO1 independent but PIEZO2 dependent, suggesting PIEZO2 (see also ${ }^{6}$ ) and not PIEZO1 as the force sensor in this situation. This was despite the fact that Yoda1, a synthetic chemical agonist of PIEZO1 but not PIEZO2, ${ }^{7}$ mimicked the effect of stretch on Tph1 expression. It led the authors to speculate about non-mechanical activators of PIEZO1 and for this they turned to the microbiome, encouraged by finding that antibiotics also reduced $5-\mathrm{HT}$ and gut motility. ${ }^{1}$ Filtered fecal solution acutely evoked intracellular $\mathrm{Ca}^{2+}$ elevations in cultured epithelial cells, as expected if a PIEZO1 activator was present. The amplitude of the response was smaller than that of Yoda1, but clearly PIEZO1 dependent. Studies of extracts from the fecal solution pointed to RNA as the active component. In support, RNase A inhibited the effect of the RNA extract and the effect of the extract was mimicked by "ssRNA40", a synthetic phosphothioate-protected single-stranded RNA (ssRNA), but not by a synthetic doublestranded RNA. Genetic disruption of PIEZO1 prevented ssRNA40 from inducing ionic current in a neuroblastoma cell line natively expressing functional PIEZO1 channels. The ssRNA40 caused 5-HT to be elevated in a PIEZO1-dependent manner and colonic infusion with RNase A reduced serum $5-\mathrm{HT}$ and gut transit time, suggesting that native ssRNA was activating PIEZO1.
This is a stimulating piece of work that covers much territory, yet the central mechanistic concept proposed is relatively simple: It is one in which, in this context, the PIEZO1 channel is not a force sensor but an ssRNA sensor. PIEZO1 is a 38-pass membrane protein that assembles in groups of 3 around a central ion pore, forming a huge triskelion force sensor and ion channel. ${ }^{4}$ Such a molecular machine may be capable of many things. In principle we already know that chemical activation is possible because Yoda1 is an agonist. ${ }^{7}$ Nevertheless, until this point in time, work in this field has suggested mechanical force as the primary natural activator of PIEZO1 channels. ${ }^{3,5}$ Even with Yoda1 it is thought that it acts by enhancing the effect of endogenous force already exerted on the channel, rather than as a stand-alone agonist. ${ }^{7}$ Sugisawa et al.'s work ${ }^{1}$ pressures us to reflect on this forcecentered view of PIEZO.

The authors argue against a role for the classical ssRNA-sensing mechanism of the endosomal Toll-like receptor 7 as an intermediate, instead envisaging separate RNA sensing in the plasma membrane with PIEZO1 at the center ${ }^{1}$ (Fig. 1a). This is a significant claim that will likely stimulate further investigations. Despite the many positives in this study, ${ }^{1}$ we await evidence that ssRNA actually binds PIEZO1 to support the authors' claim of ssRNA as a PIEZO1 ligand.

As part of these follow-up efforts, it will be interesting to further explore the relationship to PIEZO1's force-sensing capability and whether co-factors are involved. The authors' intracellular $\mathrm{Ca}^{2+}$ measurements and much of their electrophysiology data are consistent with an independent agonist effect, however, their studies of PIEZO1-knockout HEK293T cells transfected with mouse PIEZO1 surprisingly did not show basal current evoked by ssRNA but rather revealed a slowing of the inactivation rate of the mechanically-activated mouse PIEZO1 current, ${ }^{1}$ which is reminiscent of the effect of gain-of-function mutations associated with a type of anemia. ${ }^{8}$ These data suggest cooperation with the effect of mechanical force. There is also intrigue in how PIEZO2 and not PIEZO1 could mediate the stretch effect ${ }^{1,6}$ when independent head-to-head comparison has suggested similar mechanical sensitivities of these two PIEZOs. ${ }^{9}$ But the PIEZO field is nascent and we know that the native context of PIEZOs can profoundly influence their properties. ${ }^{10}$ Sugisawa et al.'s considerable efforts to deliver integrated multi-perspective insight are impressive ${ }^{1}$ and challenge us to consider new ideas.

Critical in this complex physiology is likely to be where exactly PIEZO1 is located, in which cell types and membrane compartments. Sugisawa et al.'s images of its expression in the epithelial layer ${ }^{1}$ show that the specific location is not yet entirely clear (Fig. 1b). Here, better tools and direct recordings from in situ cells will be helpful as we seek to define where this channel does its primary work.

${ }^{1}$ School of Medicine, LIGHT Building, University of Leeds, Clarendon Way, Leeds LS2 9JT, UK
Correspondence: David J. Beech (d.j.beech@leeds.ac.uk)

Published online: 3 September 2020 


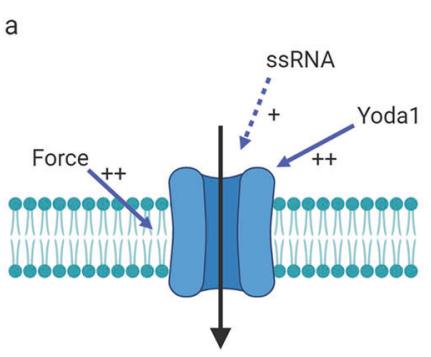

$\mathrm{Ca}^{2+}, \mathrm{Na}^{+}$ b

Intestinal lumen (feces, ssRNA, etc...)

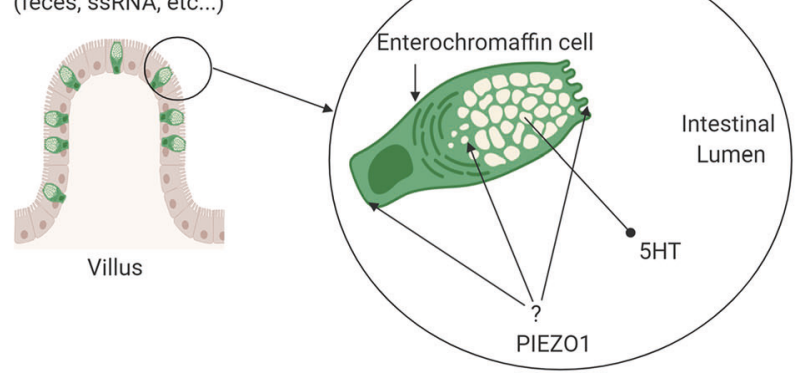

Fig. 1 PIEZO1 concepts and localization. a Simple conceptual scheme of the PIEZO1 channel with its primary known physiological activator (mechanical force), synthetic pharmacological activator (Yoda1), and the suggested new ligand and agonist (ssRNA). After activation, cellular effect is thought to result from elevated concentrations of free $\mathrm{Ca}^{2+}$ and $\mathrm{Na}^{+}$. ++ Strong activator; + weaker activator. The effects of force and Yoda1 have been confirmed by multiple independent groups and there is evidence for direct interplay with the channel, but independent confirmation and evidence for ligand interaction are understandably not yet available for ssRNA, and hence we suggest caution at this stage and use a dashed arrow. b Potential locations of PIEZO1 in the epithelial lining of the intestine (created with BioRender.com).

\section{REFERENCES}

1. Sugisawa, E. et al. Cell 182, 609-624 (2020)

2. Coste, B. et al. Science 330, 55-60 (2010).

3. Murthy, S. E., Dubin, A. E. \& Patapoutian, A. Nat. Rev. Mol. Cell Biol. 18, 771-783 (2017).

4. Saotome, K. et al. Nature 554, 481-486 (2018).

5. Beech, D. J. \& Kalli, A. C. Arterioscler. Thromb. Vasc. Biol. 39, 2228-2239 (2019).
6. Alcaino, C. et al. Proc. Natl. Acad. Sci. USA 115, E7632-E7641 (2018).

7. Syeda, R. et al. Elife 4, e07369 (2015).

8. Albuisson, J. et al. Nat. Commun. 4, 1884 (2013).

9. Poole, K., Herget, R., Lapatsina, L., Ngo, H. D. \& Lewin, G. R. Nat. Commun. 5, 3520 (2014).

10. Evans, E. L. et al. Blood 136, 140-144 (2020). 